\title{
Peri-operative electrically evoked auditory brainstem response assessment of facial nerve/ cochlea interaction at cochlear implantation
}

Nadine Schart-Morén, Karin Hallin, Sumit K. Agrawal, Hanif M. Ladak, PerOlof Eriksson, Hao Li \& Helge Rask-Andersen

To cite this article: Nadine Schart-Morén, Karin Hallin, Sumit K. Agrawal, Hanif M. Ladak, PerOlof Eriksson, Hao Li \& Helge Rask-Andersen (2018) Peri-operative electrically evoked auditory brainstem response assessment of facial nerve/cochlea interaction at cochlear implantation, Cochlear Implants International, 19:6, 324-329, DOI: 10.1080/14670100.2018.1481179

To link to this article: https://doi.org/10.1080/14670100.2018.1481179

曲 Published online: 07 Jun 2018.

Submit your article to this journal $₫$

Џ Article views: 126

View Crossmark data ¿ 


\title{
Peri-operative electrically evoked auditory brainstem response assessment of facial nerve/cochlea interaction at cochlear implantation
}

\author{
Nadine Schart-Morén ${ }^{1}$, Karin Hallin ${ }^{1}$, Sumit K. Agrawal' ${ }^{2}$, Hanif M. Ladak ${ }^{3}$, \\ Per-Olof Eriksson ${ }^{(1}$, Hao $\mathrm{Li}^{1}$, Helge Rask-Andersen ${ }^{1}{ }^{1}$
}

${ }^{1}$ Department of Surgical Sciences, Section of Otolaryngology, Head and Neck Surgery, Uppsala University Hospital, Uppsala SE-75185, Sweden, ${ }^{2}$ Department of Otolaryngology - Head and Neck Surgery, Western University, 1151 Richmond Street, London, ON, Canada, N6A3K7, ${ }^{3}$ Department of Otolaryngology - Head and Neck Surgery, Department of Medical Biophysics, Department of Electrical and Computer Engineering, Western University, 1151 Richmond Street, London, ON, Canada, N6A3K7

Objectives: Dehiscence between the cochlear otic capsule and the facial nerve canal is a rare and relatively newly described pathology. In cochlear implantation (Cl), this dehiscence may lead to adverse electric facial nerve stimulation (FNS) already at low levels, rendering its use impossible. Here, we describe an assessment technique to foresee this complication.

Methods: Pre- and postoperative computed tomography (CT) scans and intraoperative electrically evoked auditory brainstem response (e-ABR) measurements were analyzed in two patients with cochlear-facial dehiscence (CFD).

Results: Because of the relatively low resolution, the confirmation of CFD with a clinical CT was difficult. The eABR displayed a large potential with 6 and $7.5 \mathrm{~ms}$ latency, respectively, which did not occur otherwise.

Discussion: Potential strategies to resolve and manage FNS are described.

Conclusion: Prediction of FNS by assessing the distance between the labyrinthine portion of the facial nerve and the cochlea is difficult using conventional CT scans. A large evoked late myogenic potential at low stimulation levels during intraoperative e-ABR measurement may foresee FNS at $\mathrm{Cl}$ activation.

Keywords: Dehiscence, Cochlear implantation, Facial nerve stimulation, Cochlea

\section{Introduction}

The cochlea and the labyrinthine portion (LP) of the facial canal lie anatomically near one another and have been described in both histological and radioanatomical investigations. The mean distance is approximately $0.24 \mathrm{~mm}$ but ranges between 0 and $0.4 \mathrm{~mm}$ (Fang et al., 2016; Wadin and Wilbrand, 1987). The short distance may explain why electric currents can spread to the facial nerve from cochlear implant electrodes, especially in rare conditions of cochlear-facial dehiscence (CFD). This is also observed if the otic capsule is diseased like in malformations or after temporal bone fractures, or if unusual high stimulus levels are required, as in otospongiosis.

Correspondence to: Nadine Schart-Morén, Department of Otolaryngology, Head and Neck Surgery, Uppsala University Hospital, Uppsala SE-751 85, Sweden. Email: nadine.schart-moren@akademiska.se
The LP of the facial nerve runs from the internal acoustic canal to the geniculate ganglion near the upper basal turn of the cochlea (Ge and Spector, 1981; Proctor and Nager, 1982; Wadin and Wilbrand, 1987). Recently, Schart-Morén et al., (2017) used micro-computer tomography (micro-CT) with $3 \mathrm{D}$ rendering to show the extensive anatomic variations of the LP of the facial nerve (SchartMorén et al., 2017). Because of the limited resolution, a clinical CT often denies discernment of the true anatomical relationship between the cochlea and the facial nerve in cases with a suspected spread of currents to the nerve and suspected rare closeness.

Intraoperative evoked auditory brainstem response (e-ABR) measurements are routinely performed in our clinic to improve the prediction of hearing outcomes after cochlear implantation (CI) (Lundin et al., 2015). In the present study, we analyzed the possibilities of envisioning undue facial nerve stimulation 
(FNS) at device activation. Such excitation may be caused by an unusually short cochlear-facial distance. During the past year, we had two patients with FNS at our clinic during activation of the CI. The e-ABR results and the $\mathrm{CT}$ scans were thoroughly examined for both patients retrospectively. The results suggested that a small distance between the facial canal and the cochlea may be assessed preoperatively using clinical $\mathrm{CT}$ with $3 \mathrm{D}$ reconstruction, and that peri-operative e-ABR can yield a further indication of the risks for postoperative FNS.

Meanwhile, Elfarnawany et al. described improved soft-tissue visualization using SR-synchrotron radiation phase-contrast imaging (SR-PCI) (Elfarnawany et al., 2017). A plan to compare the reproduction and outcome of clinical micro-CT and SR-PCI was therefore initiated and will be published shortly. The exceptional soft-tissue resolution obtained after $3 \mathrm{D}$ reconstruction of a human cochlea with a $\mathrm{CI}$ electrode array is shown in Fig. 1.

\section{Methods}

\section{Patients}

Case 1: A 74-year-old man presented with the bilateral sensorineural hearing loss (SNHL) of unknown origin that started at the age of 15 . He suffered from bilateral severe tinnitus and hyperacusis, but had neither a balance disorder nor autophony. He had no history of otosclerosis, malformations, or head injury.

Case 2: A 71-year-old man with hereditary SNHL suffered from progressive hearing loss starting at the age of 45 . He experienced grade 1 tinnitus, but neither autophony nor balance-problems. He had no history of otosclerosis, malformations, or head injury.

\section{Cochlea implantation surgery}

The patients underwent unilateral CI surgery without complications. In both cases, FNS occurred at device activation, 4 weeks after surgery.

Case 1 was operated with a 28 -mm-long and Case 2 with a 31-mm lateral-wall electrode. Insertion was performed through the round window.

\section{Scan procedures}

Routine preoperative CT scans (Siemens Somatom Definition Flash by Siemens Healthcare, Erlangen, Germany, and slice collimation $0.3 \mathrm{~mm}$, reconstructed to $0.4 \mathrm{~mm}$ with $0.3 \mathrm{~mm}$ increments) and MRIs were assessed and showed the normal anatomy of the temporal bone and inner ear. Peri-operative X-rays showed that the electrode was fully inserted. Further investigations of the anatomical conditions were made using cone beam computer tomography (3D Accuitomo 170, J. Morita, Irvine, CA, USA, Inc.).

\section{E-ABR measurements}

E-ABR recordings were made during surgery after electrode insertion. Two separate systems were used; one for stimulation and one for recording. The stimulation system was the Med-El programing device. The recording-system was an evoked potential (EP) that was triggered from the stimulating system to initiate recording. The EP system used was the Otometrics Chartr 200 (GN Otometrics, Taastrup, Denmark). Recording-needle electrodes were placed at the vertex, forehead, and contralateral mastoid. The

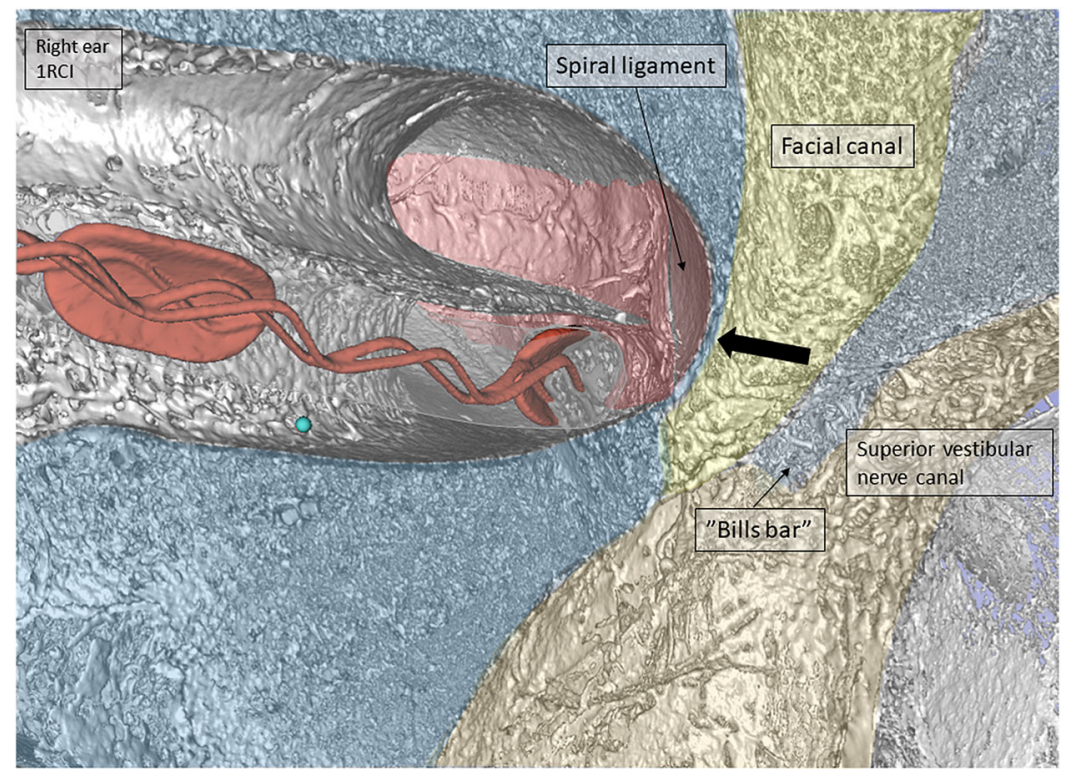

Figure 1 Synchrotron radiation phase-contrast imaging (SR-PCI) and 3D rendering of a right human temporal bone implanted with a $28 \mathrm{~mm}$ flexible lateral-wall electrode. There is a cochlear-facial juxtaposition at the upper basal turn of the cochlea corresponding to the inferior-medial aspect of the labyrinth segment of the facial nerve canal. A 3D reconstruction program was used with scalar opacity mapping thresholds to improve visualization of the surface anatomy. 
stimulation pulse width was $30 \mu \mathrm{s}$, and the current level was $1000 \mathrm{cu}$. The EP-system averaged 1000 sweeps, and the signal was filtered by a low-pass filter at $3 \mathrm{kHz}$ and a high-pass filter as $5 \mathrm{~Hz}$. EABRs were recorded from stimulating channels 1, 7, and 11 on the implant. Two separate recordings were made for each channel to verify a response.

\section{Results}

\section{E-ABR latencies and waveform}

In Case 1, intraoperative e-ABRs were recorded on channels 1, 7, and 11. Wave 5 had a low amplitude. A prominent response was observed after $7.5 \mathrm{~ms}$ (Fig. 2A). A late potential indicated a cervical muscle response. No responses were seen when stimulating channel 11. Intraoperative auditory response telemetry (ART) showed good auditory responses.

In Case 2, intraoperative e-ABRs were recorded on channels 1, 7, and 11. Stimulation on channels 1 and 7 showed uncommon late wave $\mathrm{V}$ after $5 \mathrm{~ms}$ and a larger late potential after $6 \mathrm{~ms}$ (Fig. 2B). The decayed potential was associated with side effects, such as a cervical muscle response. There was no response when stimulating channel 11. Intraoperative electrical stapedius response thresholds (e-SRT) showed vague responses but a prominent FNS at 36 qu when channel number 6 was stimulated.

\section{CT-scan}

Because of the stimulation of the facial nerve, the LP of the facial canal and its relationship to the cochlea were methodically analyzed through postoperative CBCT and 3D reconstructions (Fig. 3). Imaging results were compared to preoperative data using conventional CT images. In Case 1, the radiologist confirmed the suspicion of an anatomic dehiscence between the LP of the facial nerve and the cochlea. There was no significant difference between the ears. Electrode no. 5 was found to be in direct contact with the suspected CFD. In Case 2, the operated side showed a suspected CFD. It was harder to determine on the contralateral side because of the relatively low resolution. A suspected CFD was positioned between electrodes 5 and 6 .

The distance between the electrodes was $1.4 \mathrm{~mm}$. Because the mean length of the LP of the facial canal is usually approximately $1-2 \mathrm{~mm}$, the center of the opposing electrode may be positioned directly at the CFD, as shown in Case 1, or between two electrodes, as shown in Case 2. In both cases, electrodes 5-7 were shown to face the attenuated part of the cochlea. In both cases, this represented the first portion of the upper basal turn of the cochlea.

\section{Clinical aspects}

Case 1: During activation of the implant, 4 weeks after surgery, FNS occurred on all channels. In live mode, the eye, mouth, and neck muscles were twitching. Pulse widths were increased, but channels 4 and 5 had to be switched off. When using the processor after the pulse width was increased, the adverse facial excitation was eliminated. The stimulation levels in the map were between 11 and $16 \mathrm{qu}(1 \mathrm{qu}$ is approximately $1 \mathrm{nC}$ ), and the minimum pulse duration was set to $50 \mu$ s. Stimulation of the channels in the apical part of the cochlea created the largest problems with FNS, whereas the channels in the basal part created the fewest problems.

After six months an open speech discrimination of $16 \%$ for bisyllables was gained.

Case 2: At fitting, 4 weeks after surgery, FNS occurred on a majority of channels. Pulse widths were increased, and channels 5 and 6 had to be switched off. When wearing the processor, the situation was acceptable, but it was not possible to raise the stimulation levels further without discomfort. The stimulation levels in the map were between 19 and $27 \mathrm{qu}$, and the minimum pulse duration varied between 40 and $90 \mu \mathrm{s}$. The channels in the apical part of the cochlea were the most responsible electrodes for FNS, whereas the channels in the basal part
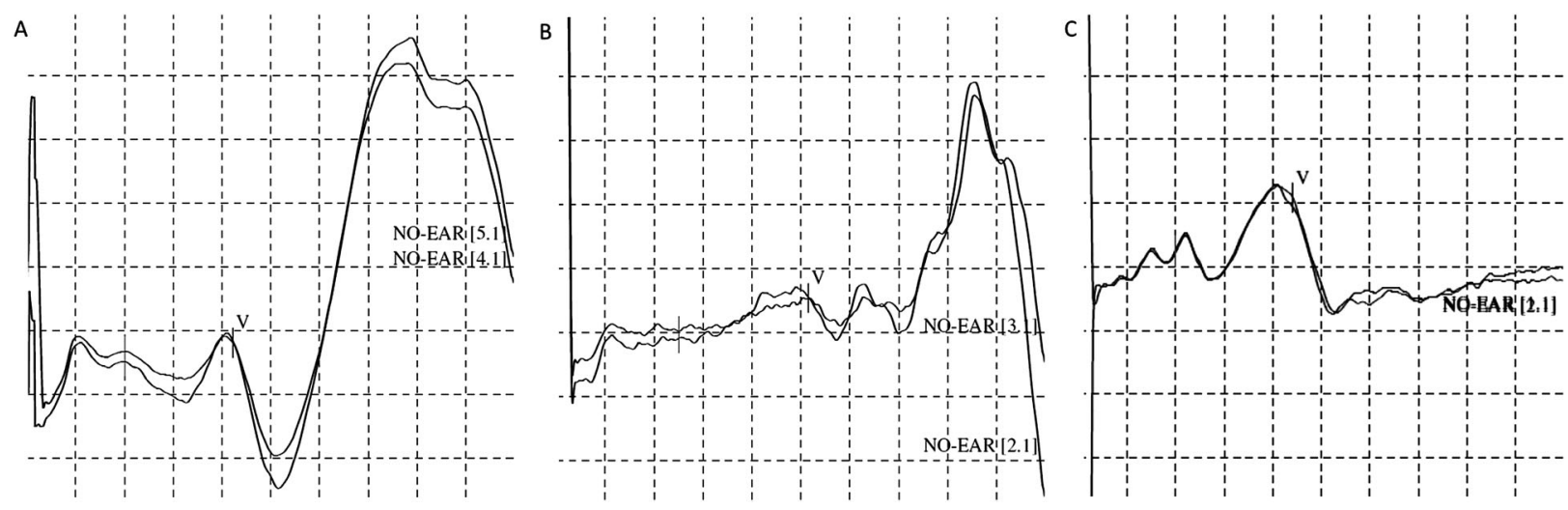

Figure 2 Illustration of peri-operative e-ABR curves. A prominent response was observed after $7.5 \mathrm{~ms}$ in case 1 (A). Uncommon late wave $V$ after $5 \mathrm{~ms}$ and a larger late potential after $6 \mathrm{~ms}$ in case 2 (B). Normal e-ABR curve (C). 


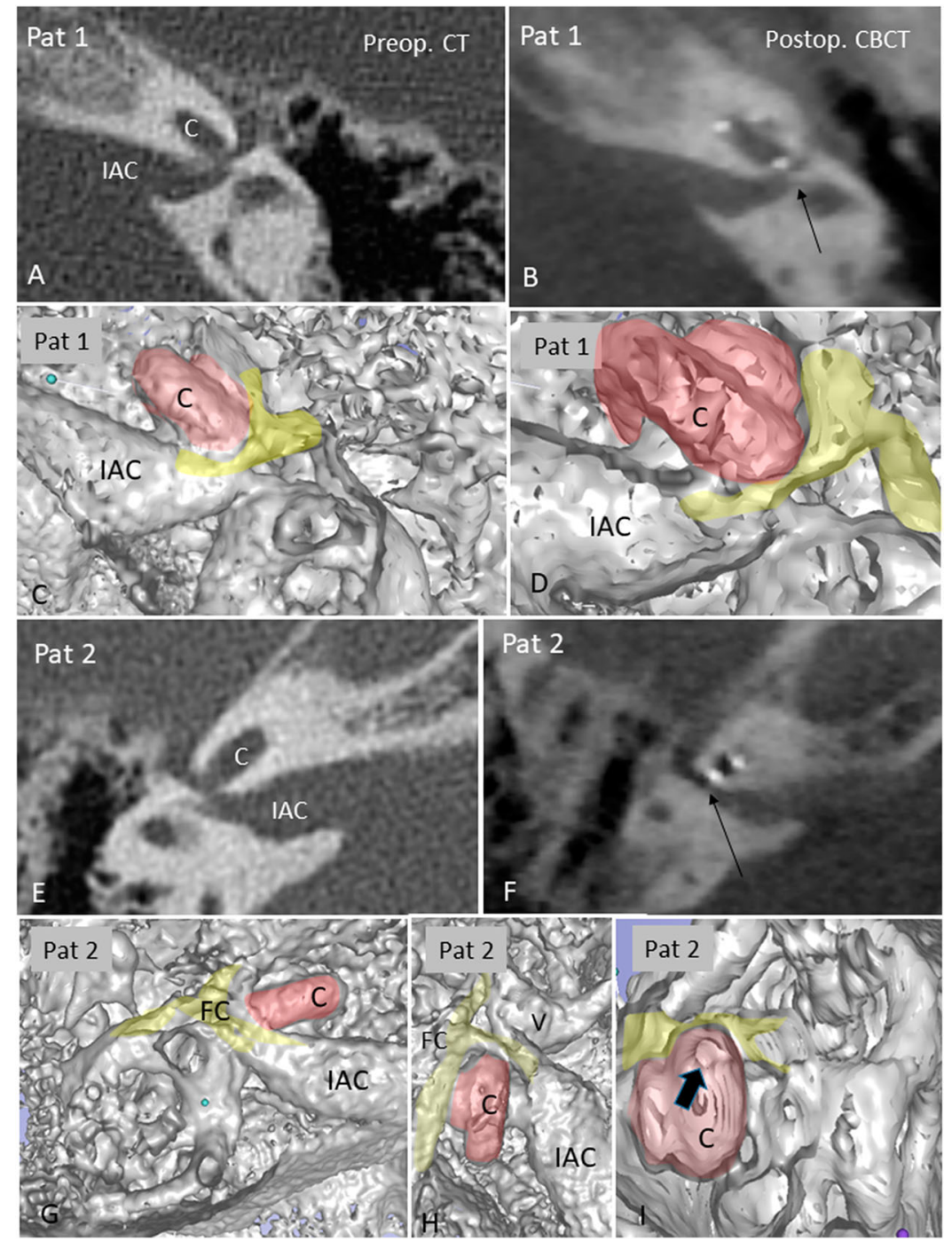

Figure 3 Preoperative (conventional) CT visualization of the anatomic interaction between the labyrinthine portion of the facial canal and the cochlea among the two patients operated with $\mathrm{Cl}$ (A and $\mathrm{E})$. Postoperative CBCT is also shown (B and $\mathrm{F}$ ) and demonstrates the position of electrodes facing the cochlea. 3D reconstruction of the temporal bone in patient 1 (C, D) and patient 2 (G-I) shows the relationship between the cochlea (C) and the facial canal (FC) in better detail. In both cases, there is a close relationship, and a dehiscence is highly suspected.

created fewer problems. After six months no open speech discrimination could be gained.

\section{Discussion}

During the past 12 months, we experienced 2 cases with CFD diagnosed after CI surgery, with excitation of the facial nerve at device activation where no other explanations, such as otosclerosis or trauma, were suspected. Blake et al. were the first to describe this clinical entity in a case report (2014). They reported two patients with hearing loss, tinnitus, and autophony. The diagnosis was further supported by a combination of history, physical examination, and high-resolution CT. CFD is a fairly rare condition.

Sahni et al. found the thin bone separation between the cochlea and the facial nerve $(0.1 \mathrm{~mm})$ in one case by micro-dissecting 60 cadaveric human temporal bones (2015). Fang et al. showed CFD in nearly $0.6 \%$ of the samples by sectioning 1020 temporal bones (2016). Later she described 3 case reports and outlines prior temporal bone irradiation as a potential risk factor for FNS (Fang et al., 2017). Our recent anatomical study using micro-CT 3D reconstructions showed considerable variations in the size and shape of the LP of the facial nerve and with CFD in 4 of 282 inner-ear molds (1.4\%) (Schart-Morén et al., 2017). Fig. 4 shows a CFD in a 3D reconstruction of a micro-CT in a fresh temporal bone.

The close relationship between the LP and the cochlea explains the incidence of FNS at CI stimulation, which varies in different conditions. It is more common in otospongiosis, malformations, and temporal bone fractures, and it may even necessitate re-implantation (Bigelow et al., 1998; Smullen et al., 2005). Electrode design seems to play an important role in the generation of FNS. Our two cases were operated with so-called lateral-wall electrodes. It may be questioned if a perimodiolar electrode should be re-implanted to improve 


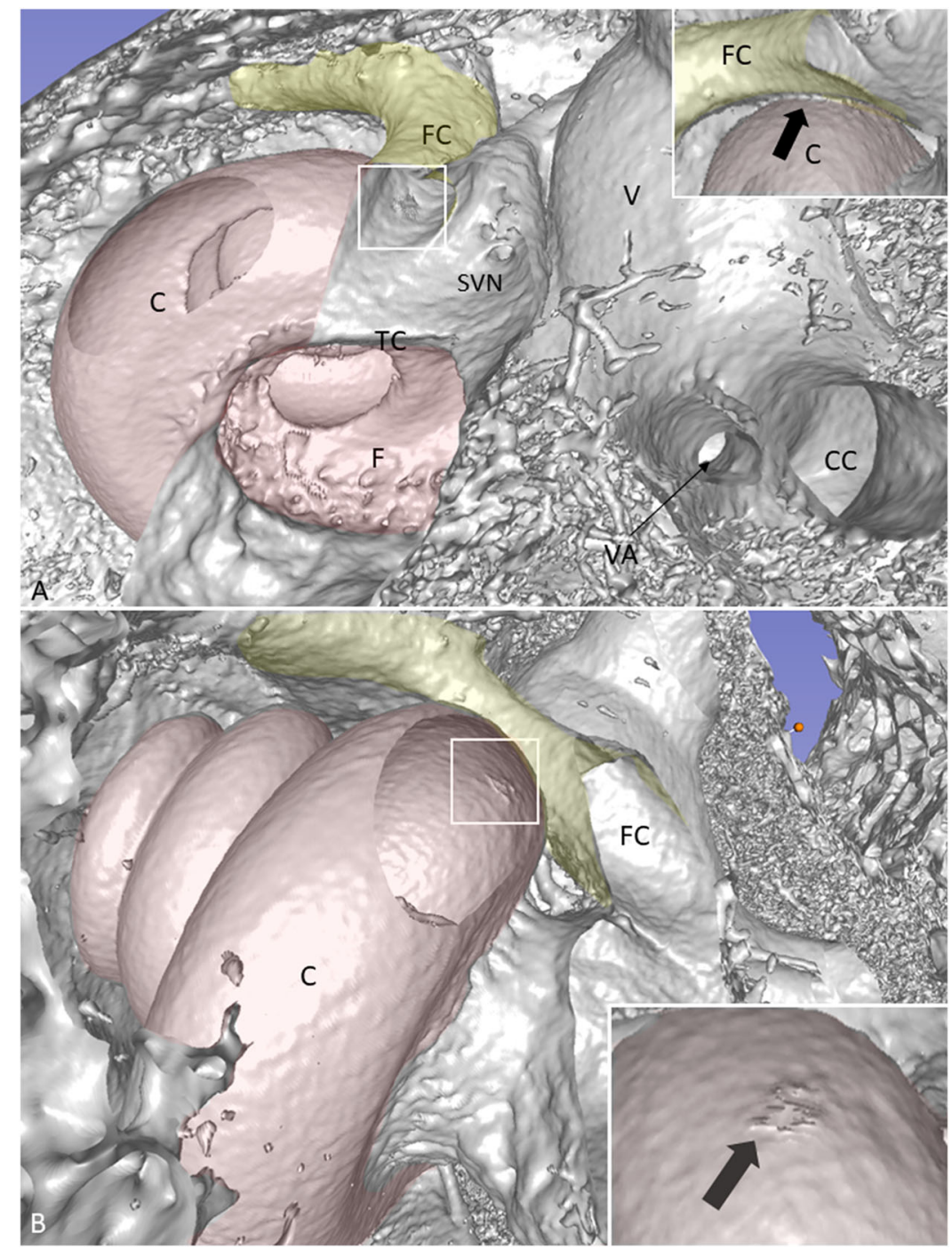

Figure 4 Micro-CT and 3D rendering of a fresh human temporal bone showing cochlea-facial dehiscence at the upper basal turn at the inferio-medial aspect of the labyrinth segment of the facial canal (right ear). A 3D reconstruction program was used with scalar opacity mapping thresholds to improve visualization of the topographic anatomy. (A) The dehiscence is observed in the facial canal. Framed area is shown in higher magnification in the inset. The cochlea and facial canal appear merged. (B) Dehiscence seen from the inside of the basal turn of the cochlea. Framed area is shown in higher magnification (bold arrow). FC: facial canal; C: cochlea; F: fundus; V: vestibule; SVN: superior vestibular nerve canal.

hearing outcome because the stimulation levels in one of the patients could not be elevated enough. A straight electrode lies near the inferior part of the spiral ligament, explaining why these electrodes may give rise to FNS more often than perimodiolar electrodes. Aschendorff et al. operated a series of 13 cases with otosclerosis and noted FNS only in the straight electrode group (2004). In otospongiosis, it may be easier to predict and prevent FNS, but a proper method to foresee CFD is lacking. Intraoperative e-ABR measurement could help in this matter.

It is known that signal contamination can occur when recording e-ABR by activating neighboring electrically excitable nonauditory structures, in particular, the facial and vestibular nerves (van den Honert and Stypulkowski, 1986). Stimulation of motor fibers in the facial nerve may result in activation of innervated muscles. Such EMG activity is often recorded prior to any visible muscle contractions and can be identified by their relatively large magnitude and steep rate of growth. Normally, a reduction in stimulus intensity reveals the underlying e-ABR waves and contrasts to the difference in magnitude between the myogenic and neurogenic potentials. In our patients, e-ABRs were measured only at one specific level $(30 \mu \mathrm{s}$, $1000 \mathrm{cu}$, which corresponds to $30 \mathrm{qu}$ ).

Before CI surgery, imaging of the temporal bone is normally performed using both clinical CT and MR for evaluation of pathologic changes and the patency of an auditory nerve. The facial canal anatomy can be visualized only partially on standard CT scans, and a CFD may easily be overseen because of the limited resolution, considering that the mean cochlear-facial distance is only $0.2 \mathrm{~mm}$. 
Fatterpekar et al. managed to present the entire course of the facial canal in 3D-volume-rendered CT images, with a quality comparable to reconstructions obtained from micro-CT data (1999). Hatch et al. showed in a recent study the difficulty in predicting FNS based solely on CF distance measurements (2017).

Triphasic stimulation, a relatively recent described method to prevent FNS was tried on Case 2 but did neither solve the problem with FNS nor increase word-recognition (Bahmer et al., 2017). With respect to the two cases, we are at least discussing re-implantation using a perimodiolar electrode in Case 2 because stimulation levels seem to not be high enough to gain open-set-word understanding.

\section{Conclusion}

Predicting FNS by measuring the distance between the LP of the facial nerve and the cochlea is difficult because of the limited resolution of conventional CT scans (distances below $0.2 \mathrm{~mm}$ ). A large evoked late myogenic potential at low stimulation levels during intraoperative e-ABR measurement may improve prediction of FNS at CI activation. It is debatable whether this may influence the surgeon's strategy to exchange an already placed lateral-wall electrode to a perimodiolar one.

\section{Disclaimer statements \\ Contributors None.}

Funding This study was supported by ALF grants from Uppsala University Hospital and Uppsala University and by the Foundation of 'Ingrid Löwenström' and kind private funds from Börje Runögård and David Giertz, Sweden.

Conflicts of interest The authors report no conflicts of interest.

Ethics approval The study was approved by Uppsala Ethical Review Board (08/23-2017, Dnr: 2017/336).

\section{ORCID}

Per-Olof Eriksson (i) http://orcid.org/0000-00031434-4771

Helge Rask-Andersen (i) http://orcid.org/0000-00022552-5001

\section{References}

Aschendorff, A., Jaekel, K., Klenzner, T., Laszig, R. 2004. Impact of electrode design on facial nerve stimulation in otosclerosis. Cochlear Implants International, 5(Suppl 1): 63-65.

Bahmer, A., Adel, Y., Baumann, U. 2017. Preventing facial nerve stimulation by triphasic pulse stimulation in cochlear implant users: intraoperative recordings. Otology \& Neurotology, 38 (10): e438-e444.

Bigelow, D.C., Kay, D.J., Rafter, K.O., Montes, M., Knox, G.W., Yousem, D.M. 1998. Facial nerve stimulation from cochlear implants. The American Journal of Otology, 19(2): 163-169.

Blake, D.M., Tomovic, S., Vazquez, A., Lee, H.J., Jyung, R.W. 2014. Cochlear-facial dehiscence - a newly described entity. The Laryngoscope, 124(1): 283-289.

Elfarnawany, M., Rohani, S.A., Ghomashchi, S., Allen, D.G., Zhu, N., Agrawal, S.K., et al. 2017. Improved middle-ear soft-tissue visualization using synchrotron radiation phase-contrast imaging. Hearing Research, 354: 1-8.

Fang, C.H., Chung, S.Y., Blake, D.M., Vazquez, A., Li, C., Carey, J.P., et al. 2016. Prevalence of cochlear-facial dehiscence in a study of 1,020 temporal bone specimens. Otology \& Neurotology, 37(7): 967-972.

Fang, C.H., Chung, S.Y., Mady, L.J., Raia, N., Lee, H.-J., Ying, Y.L.M., et al. 2017. Facial nerve stimulation outcomes after cochlear implantation with cochlear-facial dehiscence. Otolaryngology Case Reports, 3: 12-14.

Fatterpekar, G.M., Mukherji, S.K., Lin, Y, Alley, J.G., Stone, J.A., Castillo, M. 1999. Normal canals at the fundus of the internal auditory canal: CT evaluation. Journal of Computer Assisted Tomography, 23(5): 776-780.

Ge, X.X., Spector, G.J. 1981. Labyrinthine segment and geniculate ganglion of facial nerve in fetal and adult human temporal bones. The Annals of Otology, Rhinology \& Laryngology. Supplement, 90(4 Pt 2): 1-12.

Hatch, J.L., Rizk, H.G., Moore, M.W., Camposeo, E.E., Nguyen, S.A., Lambert, P.R., et al. 2017. Can preoperative CT scans be used to predict facial nerve stimulation following CI? Otology \& Neurotology, 38(8): 1112-1117.

Lundin, K., Stillesjo, F., Rask-Andersen, H. 2015. Prognostic value of electrically evoked auditory brainstem responses in cochlear implantation. Cochlear Implants International, 16(5): 254-261

Proctor, B., Nager, G.T. 1982. The facial canal: normal anatomy, variations and anomalies. I. Normal anatomy of the facial canal. The Annals of Otology, Rhinology \& Laryngology Supplement, 97: 33-44.

Sahni, D., Singla, A., Gupta, A., Gupta, T., Aggarwal, A. 2015. Relationship of cochlea with surrounding neurovascular structures and their implication in cochlear implantation. Surgical and Radiologic Anatomy, 37(8): 913---919.

Schart-Morén, N., Larsson, S., Rask-Andersen, H., Li, H. 2017. Anatomical characteristics of facial nerve and cochlea interaction. Audiology \& Neuro-otology, 22(1): 41-49.

Smullen, J.L., Polak, M., Hodges, A.V., Payne, S.B., King 3rd, J.E., Telischi, F.F., et al. 2005. Facial nerve stimulation after cochlear implantation. The Laryngoscope, 115(6): 977-982.

van den Honert, C., Stypulkowski, P.H. 1986. Characterization of the electrically evoked auditory brainstem response (ABR) in cats and humans. Hearing Research, 21(2): 109-126.

Wadin, K., Wilbrand, H. 1987. The labyrinthine portion of the facial canal. A comparative radioanatomic investigation. Acta Radiologica, 28(1): 17-23. 